\title{
OPEN Modulation of cAMP metabolism for CFTR potentiation in human airway epithelial cells
}

Jenny P. Nguyen ${ }^{1}$, Matthew Bianca ${ }^{1}$, Ryan D. Huff ${ }^{2}$, Nicholas Tiessen ${ }^{1}$, Mark D. Inman ${ }^{1} \&$ Jeremy A. Hirota ${ }^{1,2,3,4 \bowtie}$

Cystic fibrosis (CF) is a genetic disease characterized by CF transmembrane regulator (CFTR) dysfunction. With over 2000 CFTR variants identified, in addition to known patient to patient variability, there is a need for personalized treatment. The discovery of CFTR modulators has shown efficacy in certain CF populations, however there are still CF populations without valid therapeutic options. With evidence suggesting that single drug therapeutics are insufficient for optimal management of $C F$ disease, there has been an increased pursuit of combinatorial therapies. Our aim was to test cyclic AMP (CAMP) modulation, through ATP Binding Cassette Transporter C4 (ABCC4) and phosphodiesterase-4 (PDE-4) inhibition, as a potential add-on therapeutic to a clinically approved CFTR modulator, VX-770, as a method for increasing CFTR activity. Human airway epithelial cells (Calu-3) were used to test the efficacy of CAMP modulation by ABCC4 and PDE-4 inhibition through a series of concentration-response studies. Our results showed that CAMP modulation, in combination with VX-770, led to an increase in CFTR activity via an increase in sensitivity when compared to treatment of VX-770 alone. Our study suggests that CAMP modulation has potential to be pursued as an add-on therapy for the optimal management of CF disease.

Cystic fibrosis (CF) is a recessive genetic disease characterized by mutations in the gene encoding the CF transmembrane conductance regulator (CFTR), a phosphorylation-regulated ion channel responsible for conducting chloride and bicarbonate ions across epithelial cell membranes ${ }^{1-4}$. CFTR is localized at the apical membrane of epithelial cells that line primary conducting airways and submucosal glands and contributes to the regulation of airway surface lining (ASL) fluid volume and composition ${ }^{5}$. In CF, disease-causing mutations in CFTR lead to dysregulated ASL fluid volume and composition in the lung which is associated with increased mucus viscosity, increased susceptibility to pathogens, and modulated lung immunity ${ }^{6-9}$.

There are over 2000 known CFTR variants identified that are associated with a wide range of biological and functional consequences (https://www.genet.sickkids.on.ca). These variants have traditionally been classified into six classes based on their phenotype, including impairments to protein maturation, protein gating, and ion conductance ${ }^{10-12}$. However, evidence has suggested that this traditional classification is outdated since there exists CFTR variants with complex phenotypes that cannot be adequately classified into a single class ${ }^{13}$. Moreover, there has been evidence demonstrating that there are phenotypic differences between CF patients carrying the same genotypic CF variant ${ }^{14}$. This emphasizes the need for combinational therapies that will target several biological and functional defects.

Recent advancements in the molecular understanding of the functional impacts of specific CFTR variants has revolutionized drug development. As a result of discovery research focused on the function of specific CFTR variants, the Federal Drug Administration has approved four CFTR modulators ${ }^{15-18}$. The first CFTR modulator approved was CFTR potentiator ivacaftor (VX-770) which directly binds to CFTR to increase opening of the channel thus increasing chloride conductance ${ }^{19}$. In G551D-CFTR variants, VX-770 has demonstrated significant increases in predicted forced expiratory volume in $1 \mathrm{~s}\left(\mathrm{FEV}_{1}\right)$ from baseline at various doses and treatment durations ${ }^{20,21}$. VX-770 was subsequently assessed in combination with the CFTR corrector lumacaftor (VX-809), which increases the amount of CFTR reaching the cell surface, and showed mild improvements in FEV (2.6-4 $_{1}$ percent points) and decreases in pulmonary exacerbations (30-39\%) in the F508del homozygous population,

${ }^{1}$ Division of Respirology, Department of Medicine, Firestone Institute for Respiratory Health, McMaster University, Hamilton, ON L8N 4A6, Canada. ${ }^{2}$ Division of Respiratory Medicine, Department of Medicine, University of British Columbia, Vancouver, BC V6H 3Z6, Canada. ${ }^{3}$ McMaster Immunology Research Centre, McMaster University, Hamilton, ON L8S 4K1, Canada. "Department of Biology, University of Waterloo, Waterloo, ON N2L 3G1, Canada. ${ }^{\boxplus}$ email: hirotaja@mcmaster.ca 
which represents the global majority of CF patients ${ }^{22-24}$. Additional CFTR modulators approved include CFTR correctors tezacaftor (VX-661) and elexacaftor (VX-445) ${ }^{25-28}$. On-going combinatorial studies with VX-770, novel potentiators, VX-561 - which is an altered form of VX-770, ABBV-3067, and PTI-808; novel correctors, VX-661, VX-445, VX-659, VX-121, ABBV-2222, and PTI-801; and amplifier, PTI-428, which increases the amount of CFTR produced, suggest that a single drug therapy will not be sufficient for optimal management of CF disease (NCT03912233, NCT03969888, NCT03500263) ${ }^{25-30}$. Furthermore, although combinatorial CFTR modulator therapies have shown efficacy in previously poorly managed CF populations, many CFTR variants have not been studied at an individual level. In addition, these CFTR modulator therapies directly target CFTR defects and do not take into account mechanisms implicated in CFTR activity, such as phosphorylation of the regulatory domain of $\mathrm{CFTR}^{31-33}$. As such, new drugs and drug combinations that align with precision medicine approaches should be developed to ensure that there are valid therapeutic options for each individual with CF.

While understanding the genetic variation in CF patients is important for developing combinatorial therapies, there are many factors that can influence CFTR potentiation, including cyclic AMP (cAMP) metabolism. CFTR is phosphorylated by the cAMP-dependent enzyme protein kinase A (PKA) $)^{31,32}$. Following a rise in intracellular cAMP, PKA is activated and can phosphorylate CFTR, increasing open channel probability ${ }^{31-33}$. There are multiple mechanisms contributing to cAMP regulation, including CAMP-efflux transporters, phosphodiesterases (PDE), and $\beta_{2}$ adrenergic receptors ${ }^{14,34-39}$. A key cAMP transporter is ATP Binding Cassette Transporter C4 (ABCC4), formerly known as multi-drug resistance protein $-4^{34,40}$. ABCC4 is functionally and physically coupled with CFTR through the scaffolding protein PDZK1 ${ }^{34}$. Inhibition of ABCC4 with MK-571 was first shown to be able to attenuate cAMP transport and potentiate CFTR activity in gut epithelial cells, however this has been later demonstrated in airway epithelial cells ${ }^{14,34,41,42}$. In the context of CF, we have demonstrated that pharmacological inhibition of ABCC4 in human airway epithelial cells is able to potentiate CFTR in G551D-variants above and beyond VX-770 14 . Aside from ABCC4 inhibition, there has also been evidence demonstrating that pharmacological inhibition of PDE- 4 was able to potentiate CFTR activity through the elevation of cAMP levels ${ }^{37,38,43}$. The interaction of ABCC4 and PDE-4 inhibition to increase CAMP levels to augment CFTR potentiation remains to be explored.

Currently there are several investigational or clinically available therapeutics that have potential applications in influencing CFTR activity through cAMP elevation. MK-571 has been previously used as an ABCC4 inhibitor to prevent cAMP-efflux, resulting in an increase in CFTR activity in human airway epithelial cells ${ }^{14,41,42}$. MK-571 was designed as a cysteinyl leukotriene receptor antagonist and is exploited as a research compound due to off-target effects that include ABCC4 and PDE-4 inhibition and has also been used in humans safely ${ }^{44}$. Conversely, Ceefourin-1 and 2 have been reported as ABCC4 inhibitors with demonstrated in vitro efficacy and selectivity ${ }^{45}$. Roflumilast is a clinically available selective PDE-4 inhibitor with anti-inflammatory effects that is commonly administered to chronic obstructive pulmonary disease (COPD) patients suffering from chronic bronchitis $^{46-49}$. Similar to Roflumilast, Rolipram is also known to be a selective PDE-4 inhibitor. Originally it was investigated as a potential antidepressant drug, however, it is not used clinically due to adverse side-effects and its small therapeutic window ${ }^{50-52}$.

Due to our previous demonstration that pharmacological inhibition of ABCC4, in the presence of CFTR modulator VX-770, leads to CFTR potentiation beyond VX-770 alone and evidence demonstrating the use of PDE-4 inhibitors is also able to potentiate CFTR activity, we hypothesize that cAMP modulation with ABCC4 and PDE-4 inhibitors, in the presence of VX-770, will potentiate CFTR activity. In order to begin defining the efficacy of cAMP modulation by ABCC4 and PDE-4 inhibitors on CFTR activity, we pursued a series of concentration-response studies on Calu-3 human airway epithelial cells with wild-type CFTR ${ }^{53-55}$. We demonstrate that combinatorial additions of CFTR modulator VX-770 with either an ABCC4 or PDE-4 inhibitor led to CFTR potentiation via an increase in sensitivity. Our results suggest that combinatorial additions of VX-770 with ABCC4 or PDE-4 inhibitors may increase sensitivity and efficacy of VX-770 alone.

\section{Results}

In vitro extracellular CAMP assay analysis of ABCC4 inhibitor compounds in human airway epithelial cells. An in vitro extracellular cAMP assay of two commercially available ABCC4 inhibitors, MK-571 and Ceefourin-1 (Fig. 1a,b), was performed in human airway epithelial Calu-3 cells, and human bronchial epithelial cells (HBEC6-KT, Supplementary Fig. 1), to define their efficacy in decreasing extracellular cAMP levels. Concentration-dependent ABCC4 inhibition using MK-571 and Ceefourin-1 (Fig. 1) caused a decrease in extracellular cAMP levels in both cell lines. The half maximal inhibitory concentration $\left(\mathrm{IC}_{50}\right)$ values of MK-571 and Ceefourin-1 were found to be $2.6 \mu \mathrm{M}$ and $0.7 \mu \mathrm{M}$ respectively and defined a role for ABCC4-mediated cAMP transport in human airway epithelial cells that may be leveraged for CFTR potentiation.

Detection of ABCC4 and CFTR in human airway epithelial cell lines. The expression and function of ABCC4 has been confirmed in HBEC6-KT cells (Supplementary Fig. 1) although CFTR expression and function remain to be defined ${ }^{41,42}$. In contrast, human airway epithelial Calu-3 cells are well documented for CFTR expression and function but not for ABCC $4^{53-55}$.

ABCC4 and CFTR protein expression levels were therefore probed via immunoblot in HBEC6-KT and Calu-3 cell lines with total protein stain performed as a loading control (Fig. 2). Due to reported time-dependent differentiation and polarization of Calu- 3 cells, protein was extracted at 4 different time points $(0,7,14$, and 21 days post-confluency $)^{53,56-58}$. ABCC4 was present in both HBEC6-KT and Calu-3 cells at all-time points, indicated by the bands present at $150 \mathrm{kDa}$. CFTR was present at all-time points in Calu-3 cells. No CFTR was detected in HBEC6-KT cells. Due to co-expression of ABCC4 and CFTR, Calu-3 cells were used for subsequent studies exploring the interrelationships between ABCC4, cAMP modulation, and CFTR. 

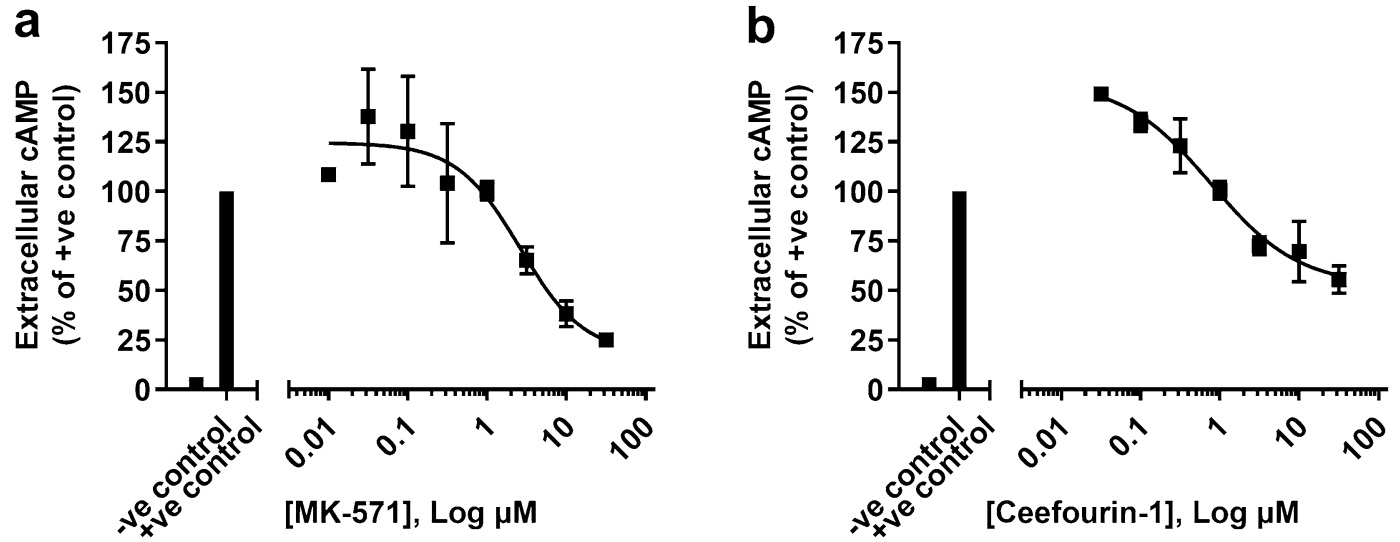

Figure 1. Concentration-response analysis of compounds with ABCC4 inhibition properties on extracellular cAMP from human airway epithelial cells. Human airway epithelial (Calu-3) cells were pre-treated with IBMX $(20 \mu \mathrm{M})$, exposed to (a) MK-571, (b) Ceefourin-1, or DMSO vehicle control, and then treated with forskolin $(10 \mu \mathrm{M})$. Cell culture supernatants were assessed for cAMP levels $24 \mathrm{~h}$ post-treatment. Each concentrationresponse curve was normalized to positive control (IBMX + forskolin) with data presented as means \pm standard deviations ( $n=3$, MK-571; $n=3$, Ceefourin-1).

a

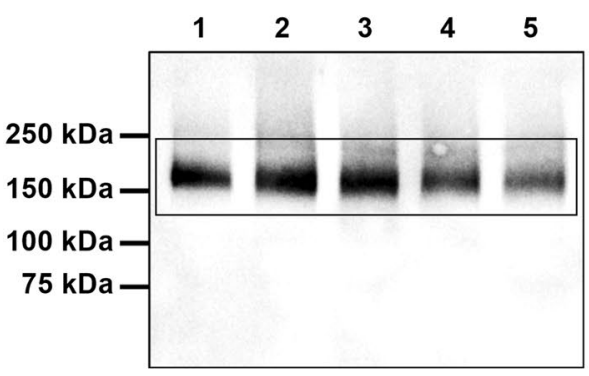

b

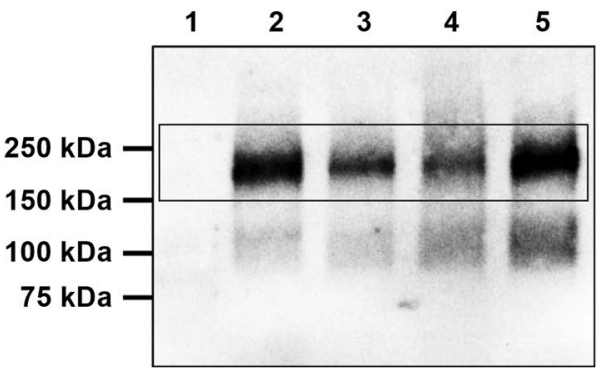

d

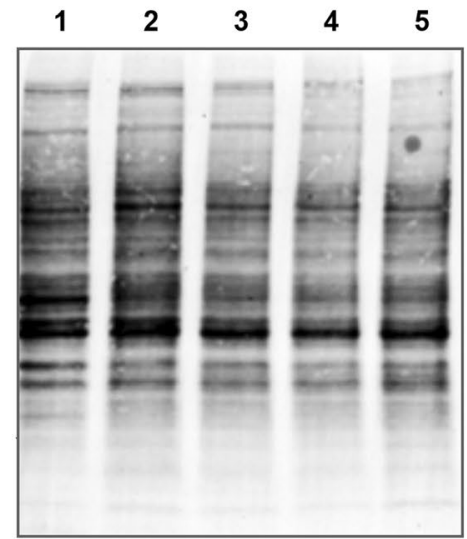

Lane 1: HBEC6-KT

Lane 2: Calu-3, 0 Days P.C. Lane 3: Calu-3, 7 Days P.C. Lane 4: Calu-3, 14 Days P.C. Lane 5: Calu-3, 21 Days P.C.
C

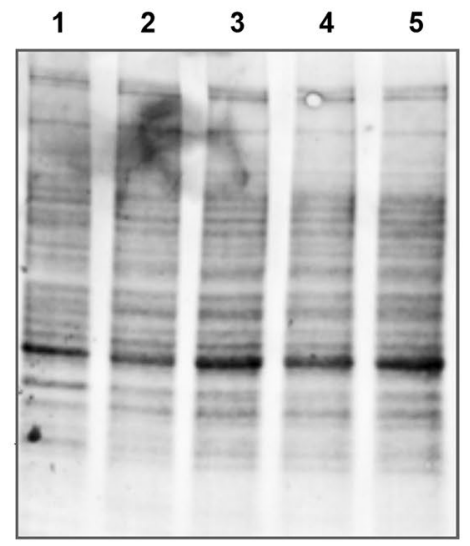

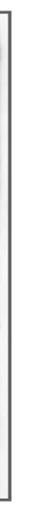

Figure 2. Characterization of ABCC4 and CFTR protein expression in two human airway epithelial cell lines. (a) ABCC4 and (b) CFTR in both HBEC6-KT and Calu-3 cells. For Calu-3 cells, extracts were retrieved at several different time points post-confluence (P.C.). (a) ABCC4 was detected in both HBEC6-KT and Calu-3 cells and (b) CFTR was only detected in Calu-3 cells. A total protein stain was performed as the loading control for (c) ABCC4 and (d) CFTR blots. Full-length blots are presented in Supplementary Fig. 4. 


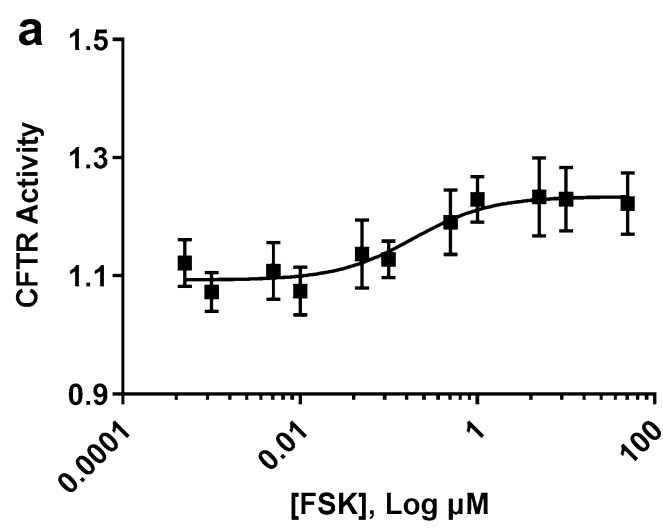

EC50 $=0.19$

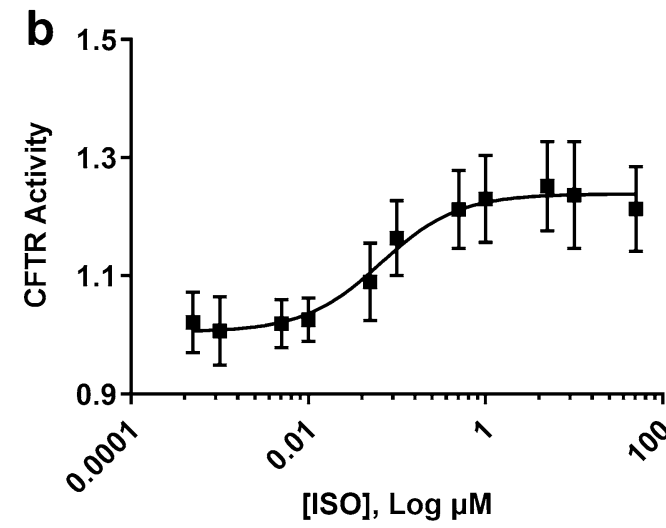

EC50 $=0.066$

Figure 3. Concentration-response analysis of two different cAMP-elevating agents on CFTR activity. Calu-3 cells were exposed to G protein-coupled receptor-independent cAMP inducer (a) forskolin and G proteincoupled receptor-dependent cAMP inducer (b) isoproterenol at increasing concentrations while measuring CFTR activity using an in vitro CFTR membrane potential assay. $\mathrm{EC}_{50}$ values were analyzed and reported. Each concentration-response curve was normalized to baseline over DMSO vehicle control with data presented as \pm standard deviations $(n=8)$.

Receptor-dependent and -independent activation of CFTR activity-impact of VX-770. A concentration-response analysis was performed with forskolin (FSK) and isoproterenol (ISO), G protein-coupled receptor-independent and -dependent cAMP inducers, respectively (Fig. 3). The half maximal effective concentration $\left(\mathrm{EC}_{50}\right)$ value for FSK and ISO were determined to be $0.19 \mu \mathrm{M}$ and $0.07 \mu \mathrm{M}$ respectively, with both compounds demonstrating ability to induce CFTR activity as measured by a validated membrane potential sensitive assay ${ }^{14}$.

Concentration-response curves with CFTR modulator VX-770, using both receptor-independent FSK and receptor-dependent ISO cAMP inducers (Fig. 4a,e), demonstrated an upward and leftward shift in the curve, indicating an increase in CFTR activity. Area under the curve (AUC) analysis showed a significant increase with the addition of VX-770 with either cAMP inducer (Fig. 4b,f; ${ }^{* *} P \leq 0.01$ and ${ }^{* * *} P \leq 0.0001$ ). VX-770 increased the sensitivity of FSK-induced CFTR activity as measured by $\mathrm{EC}_{50}$ analysis (Fig. $4 \mathrm{c} ;{ }^{\star \star} P \leq 0.01$ ). However, the maximal effect concentration $\left(\mathrm{E}_{\max }\right)$ remained unchanged (Fig. $4 \mathrm{~d}$ ). Despite changes in AUC, no changes in $\mathrm{EC}_{50}$ or $\mathrm{E}_{\max }$ were observed with the combination of VX-770 and ISO (Fig. 4g,h).

Collectively, these results demonstrate that both receptor-dependent and -independent mechanisms of cAMP elevation are able to induce CFTR activity in Calu-3 cells, which in turn is potentiated with VX-770.

Effect of ABCC4 and PDE-4 inhibition on CFTR activity. Following our quantification of CFTR activity with the clinically approved CFTR potentiator, VX-770, we next investigated the effect of ABCC4 inhibition and PDE-4 inhibition in Calu-3 cells.

For pharmacological inhibition of ABCC4, we used MK-571 and Ceefourin-1 (see Fig. 1) with FSK and ISO (Fig. 5a-d). No combination of ABCC4 inhibitor and cAMP-elevating agent changed CFTR activity in Calu-3 cells when examining AUC, $\mathrm{EC}_{50}, \mathrm{E}_{\max }$, and baseline values (Fig. 5e-h).

For pharmacological inhibition of PDE-4 we used Roflumilast and Rolipram with FSK and ISO (Fig. 6a-d). In contrast to ABCC4 inhibition, the concentration-response curves of PDE-4 inhibitors showed leftward shifts with ISO (Fig. 6c,d). Similarly, AUC analysis revealed significant increases with the addition of either Roflumilast or Rolipram with ISO but not FSK (Fig. $6 \mathrm{e} ;{ }^{\star} P \leq 0.05$ ). $\mathrm{EC}_{50}, \mathrm{E}_{\mathrm{max}}$, and baseline values for PDE-4 inhibitors with either cAMP inducer were not different (Fig. 6f-h). In addition to Roflumilast and Rolipram, pharmacological inhibition using non-specific PDE inhibitor IBMX was also tested (Supplementary Fig. 2). Significant increases in AUC (Supplementary Fig. $2 c, i ;{ }^{\star} P \leq 0.05 ;{ }^{*} P \leq 0.01$ ), with either cAMP inducer, were seen and significant increases in baseline values for IBMX with ISO only was revealed (Supplementary Fig. $21 ;{ }^{\star} P \leq 0.05$ ).

Effect of CAMP modulation with VX-770 on CFTR activity. Due to our observations that ABCC4 and PDE-4 inhibition alone showed minor potentiation of CFTR activity relative to VX-770, we next investigated whether a combinatorial approach could be more efficacious ${ }^{13}$.

Concentration-response curves of VX-770 + MK-571 or Roflumilast combinations with FSK stimulation led to an upward shift of the curve at all concentrations, indicating an increase in CFTR activity (Fig. 7a,b). VX-770 + MK-571 or Roflumilast combinations with ISO also led to shifts in the curves, however only at lower concentrations (Fig. 7c,d). For the AUC analysis of each combination, increases were observed with FSK but not ISO (Fig. $7 \mathrm{e} ;{ }^{\star \star} P \leq 0.01$, and ${ }^{* *} P \leq 0.001$ ). $\mathrm{EC}_{50}$ and $\mathrm{E}_{\max }$ analysis for each combination with both cAMP inducers showed no difference (Fig. $7 f, g$ ). 
a

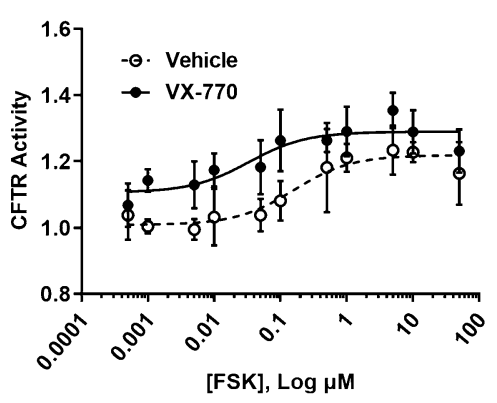

e

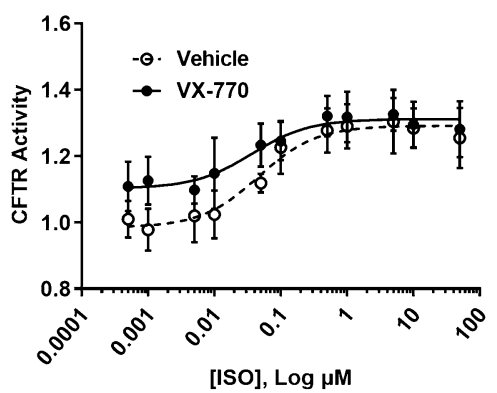

b

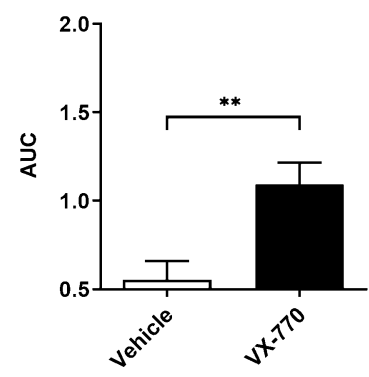

f

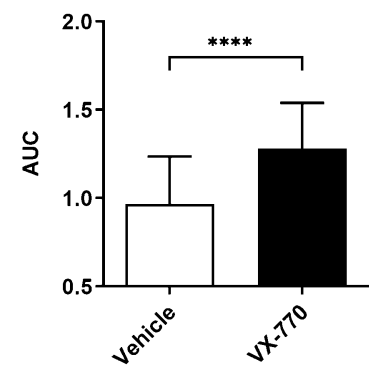

C

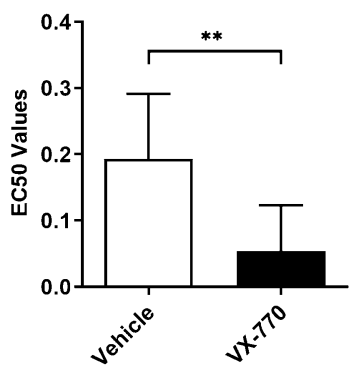

g

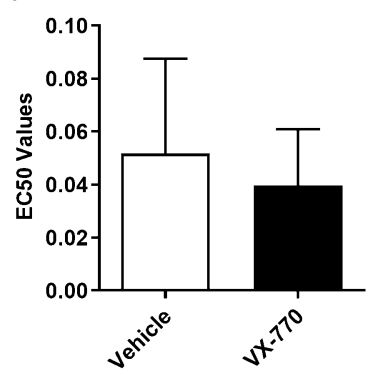

d

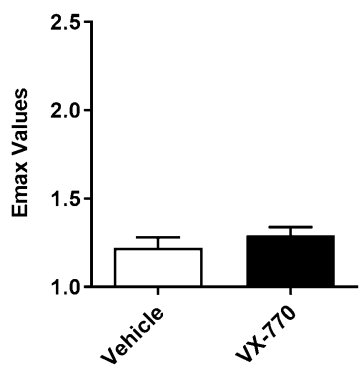

h

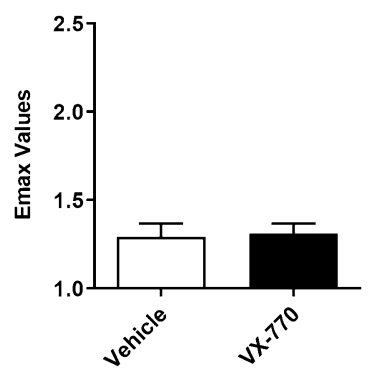

Figure 4. Consequences of CFTR modulator VX-770 on CFTR function using receptor-independent (forskolin) and receptor-dependent (isoproterenol) cAMP inducers. Calu-3 cells were stimulated with VX-770 $(1 \mu \mathrm{M})$ in the presence of cAMP inducer (a) forskolin and (e) isoproterenol. Analysis of concentration-response curves was performed for $(\mathbf{b}, \mathbf{f}) \mathrm{AUC},(\mathbf{c}, \mathbf{g}) \mathrm{EC}_{50}$, and $(\mathbf{d}, \mathbf{h}) \mathrm{E}_{\max }$. The concentration-response curve was normalized to baseline over DMSO vehicle control. All data presented as \pm standard deviations $(n=4, F S K ; n=8$, ISO). ${ }^{* *} P \leq 0.01 ;{ }^{* * * *} P \leq 0.0001$.

The consequences of these pharmacological intervention combinations with FSK, in addition to Roflumilast + MK-571 + VX-770 combinations, were also tested in primary human bronchial epithelial cells (HBECs), from two independent donors, grown under air-liquid interface culture conditions (Supplementary Fig. 3). In one donor sample, significant increases in AUC and max peak analysis, beyond VX-770 alone were observed. For AUC, Roflumilast \pm VX-770, Roflumilast + MK-571, and Roflumilast + MK-571 + VX-770 combinations were significantly greater than VX-770 alone (Supplementary Fig. $3 c ;{ }^{* *} P \leq 0.001 ;{ }^{* * *} P \leq 0.0001$ ). Similarly, the max peak achieved with Roflumilast \pm VX-770, MK-571 + VX-770, Roflumilast + MK-571, and Roflumilast + MK-571 + VX-770 were significantly greater than VX-770 alone as well (Supplementary Fig. $3 \mathrm{~d} ;{ }^{*} P \leq 0.05$; $\left.{ }^{* * * *} P \leq 0.0001\right)$. These effects were not observed in the other donor sample (Supplementary Fig. 3g,h).

To compare our series of cAMP modulation interventions with and without VX-770 (Figs. 4, 5, 6, 7), foldchange comparisons of $\mathrm{AUC}, \mathrm{EC}_{50}$, and $\mathrm{E}_{\max }$, relative to control conditions were performed for all experiments (Fig. 8). Fold-change analysis of AUC with FSK stimulation showed that VX-770 was superior to either ABCC4 (MK-571) or PDE-4 (Roflumilast) inhibition alone, and that combinatorial approaches did not potentiate CFTR AUC values (Fig. $8 \mathrm{a},{ }^{* *} P \leq 0.01$ and ${ }^{* *} P \leq 0.001$ ). ISO stimulation resulted in similar modest trends that were not significant (Fig. 8d). Fold-change EC $_{50}$ analysis between VX-770 alone to VX-770 with ABCC4 or PDE-4 inhibition showed a significant decrease with ISO for both combinatorial approaches, observations that were absent with FSK stimulation. (Fig. 8b,e, ${ }^{\star} P \leq 0.05$ ). Lastly, fold-change $\mathrm{E}_{\max }$ analysis performed with either FSK or ISO showed no changes for any combinatorial approach (Fig. 8c,f).

\section{Discussion}

There is an increased need for combinatorial therapies for CF subjects to optimally treat all subjects at a personalized level ${ }^{13,25,27,29}$. CFTR potentiation with VX-770 represents a high-water mark for increased chloride conductance in CF patients. In the present study, we quantified the capacity of combinatorial approaches that modulate intracellular cAMP levels to activate CFTR. Specifically, we inhibited ABCC4, a cAMP-efflux pathway, and PDE-4, a cAMP metabolism pathway. Using Calu-3 cells, we demonstrate that interventions targeting intracellular CAMP are unable to potentiate CFTR to levels observed with VX-770. Combinatorial approaches of cAMP modulation with VX-770 suggest that increases in CFTR activity as measured by AUC and EC ${ }_{50}$ values, are possible and warrant further exploration in primary human airway epithelial cells expressing loss of function CFTR variants ${ }^{14}$.

The addition of VX-770 to Calu-3 cells induced shifts in the curve with both FSK and ISO, providing context for the amount of CFTR potentiation possible in Calu-3 cells with a clinically approved CFTR potentiator. While there was a significant change to $\mathrm{EC}_{50}$ with FSK, no changes were seen with ISO, suggesting that mechanisms 
a

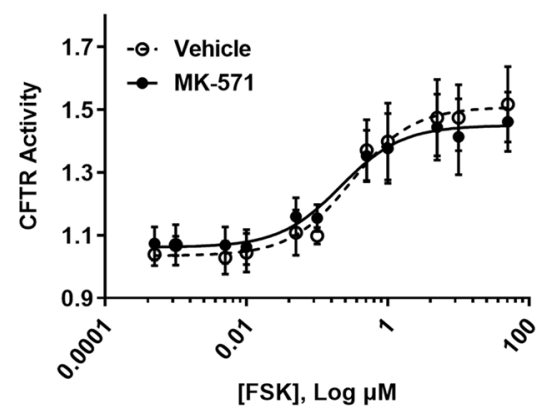

C

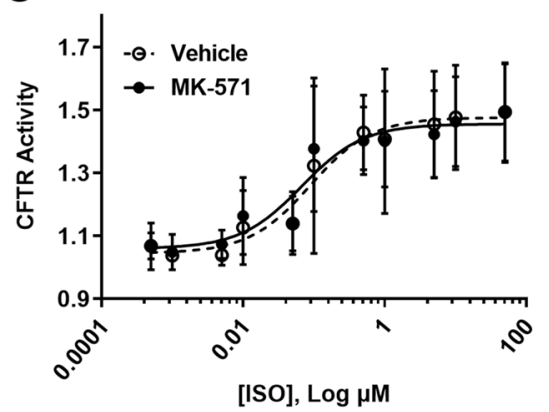

e

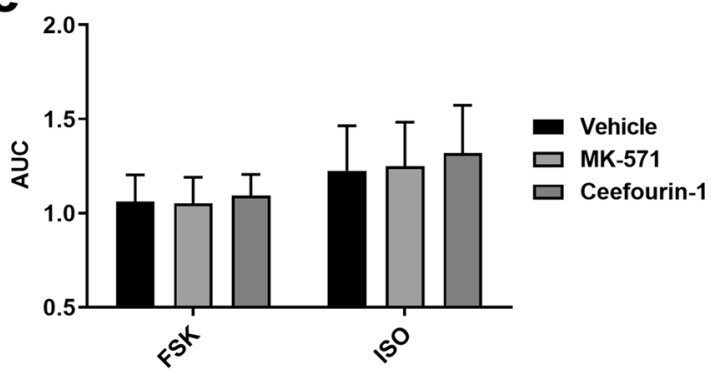

g

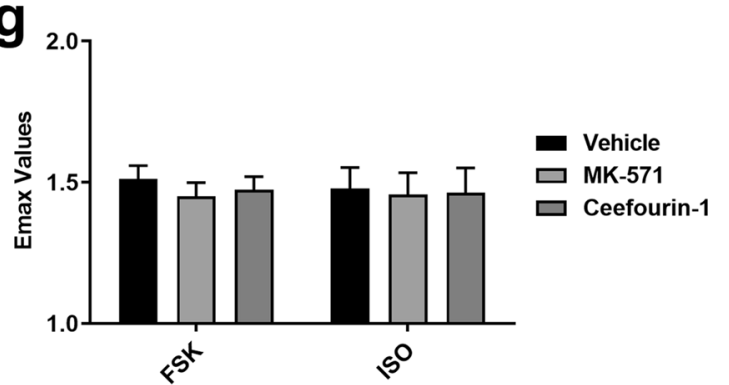

b

Ceefourin-1

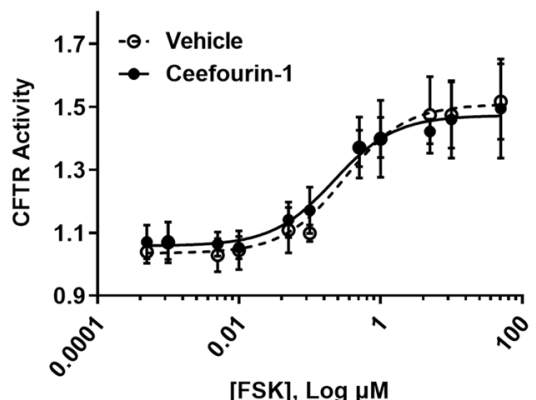

d

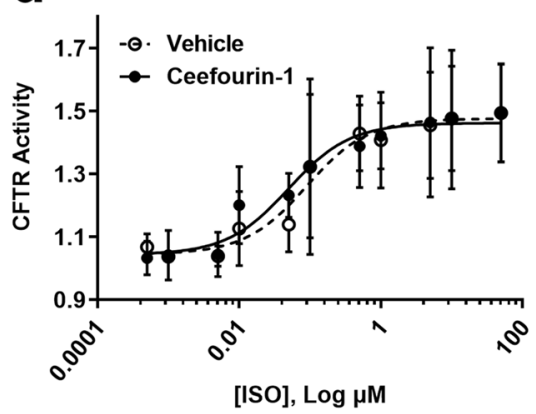

f
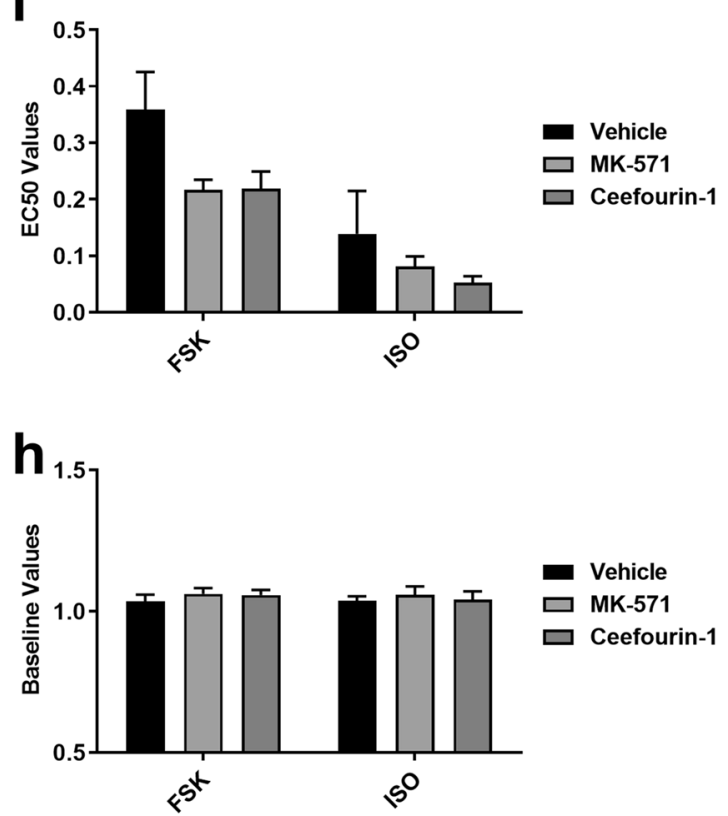

Figure 5. Consequences of ABCC4 inhibition on CFTR function using receptor-independent (forskolin) and receptor-dependent (isoproterenol) cAMP inducers. ABCC4 was inhibited with MK-571 and Ceefourin-1 using submaximal concentrations determined from extracellular cAMP assays. Forskolin-stimulated (a,b) CFTR activity in the presence of (a) MK-571 (1.8 $\mu \mathrm{M})$ and (b) Ceefourin-1 (4.8 $\mu \mathrm{M})$. Isoproterenol-stimulated (c,d) CFTR activity in the presence of (c) MK-571 and (d) Ceefourin-1. Analysis of concentration-response curves was performed for (e) AUC, (f) $\mathrm{EC}_{50},(\mathbf{g}) \mathrm{E}_{\max }$, and (h) Baseline. Each concentration-response curve was normalized to baseline over DMSO vehicle control. All data presented as means \pm standard deviations $(\mathrm{n}=5$, FSK; $\mathrm{n}=4$, ISO).

governing cAMP elevations may influence downstream analyses of CFTR activities ${ }^{59-63}$. For both cAMP inducers, the $\mathrm{E}_{\max }$ values were not significant and while there were no significant changes to baseline with FSK, there was a 
a

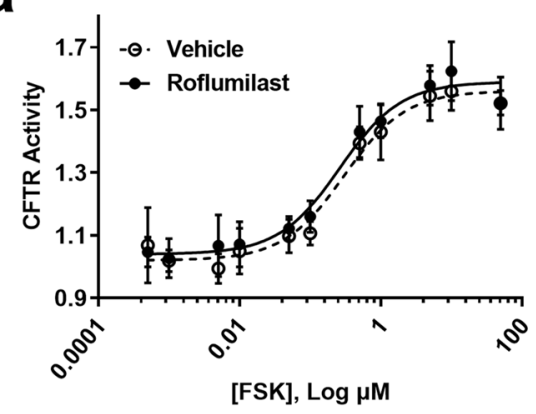

C

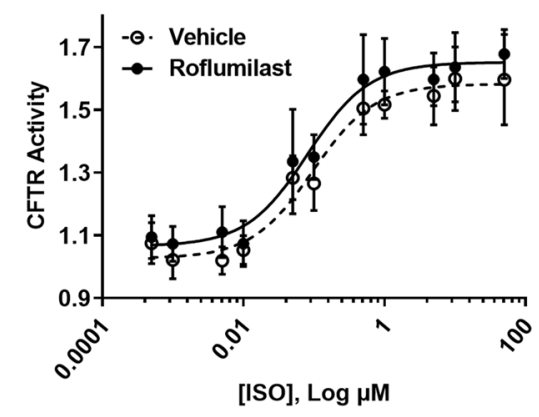

e

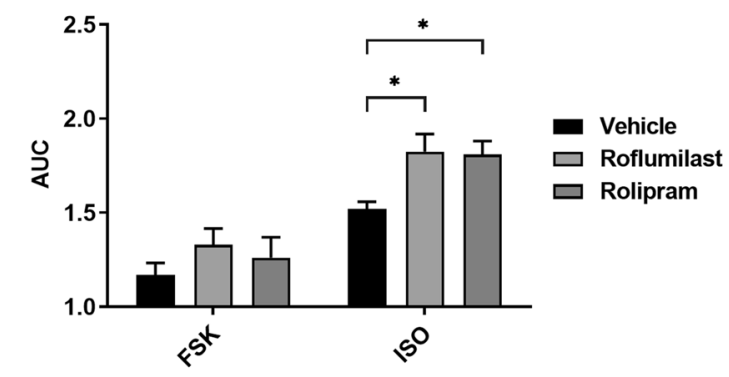

g

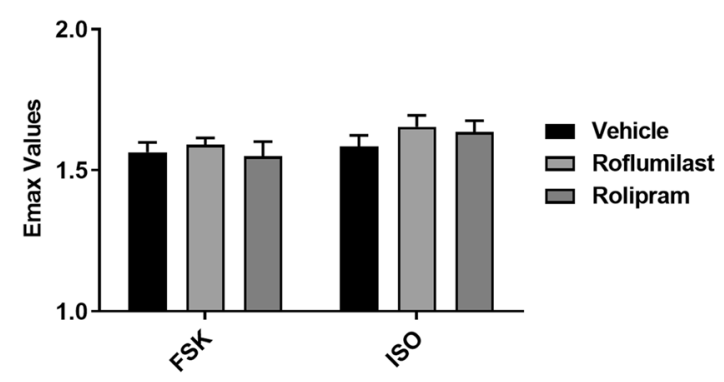

b

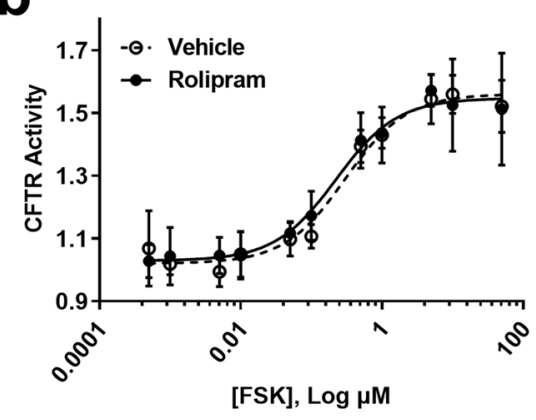

d

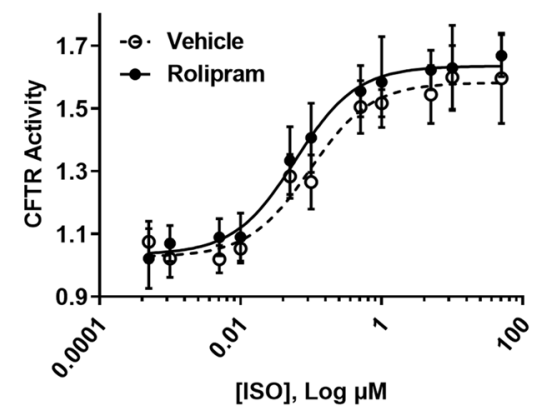

f

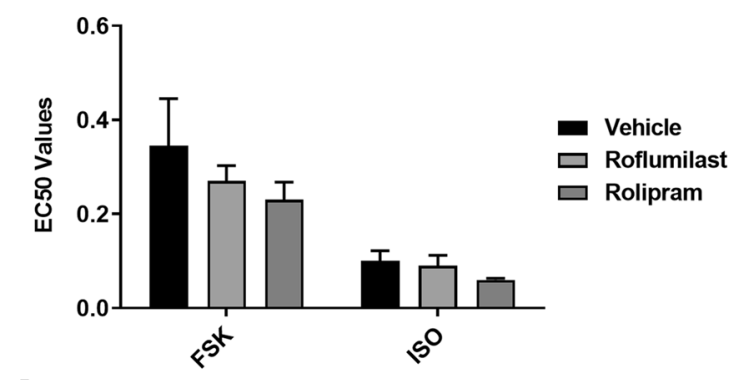

h

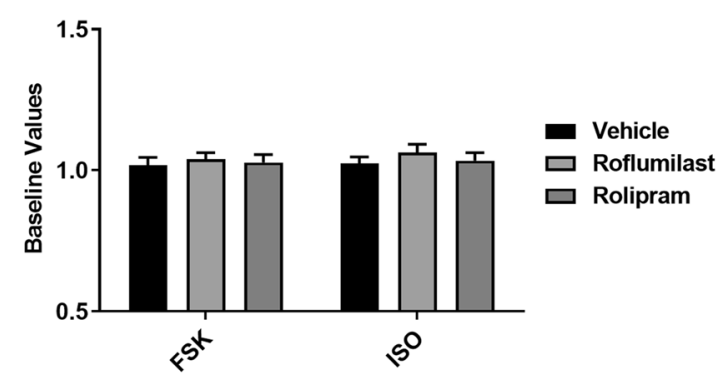

Figure 6. Consequences of PDE-4 inhibition on CFTR function using receptor-independent (forskolin) and receptor-dependent (isoproterenol) cAMP inducers. PDE-4 was inhibited with Roflumilast and Rolipram at submaximal concentrations. Forskolin-stimulated (a,b) CFTR activity in the presence of (a) Roflumilast $(1 \mu \mathrm{M})$ and (b) Rolipram $(10 \mu \mathrm{M})$. Isoproterenol-stimulated (c,d) CFTR activity in the presence of (c) Roflumilast and (d) Rolipram. Analysis of concentration-response curves was performed for (e) AUC, (f) $\mathrm{EC}_{50},(\mathbf{g}) \mathrm{E}_{\max }$, and (h) Baseline. Each concentration-response curve was normalized to baseline over DMSO vehicle control. All data presented as means \pm standard deviations $(n=5) .{ }^{\star} P \leq 0.05$.

significant increase in baseline with ISO (data not shown). Collectively, this suggests not only is the mechanism of action in elevating cAMP levels affecting sensitivity, but also efficacy ${ }^{59-63}$. Collectively, these results suggest that it is possible to potentiate CFTR activity in Calu-3 cells by modulating cAMP levels. 
MK-571 + VX-770

a

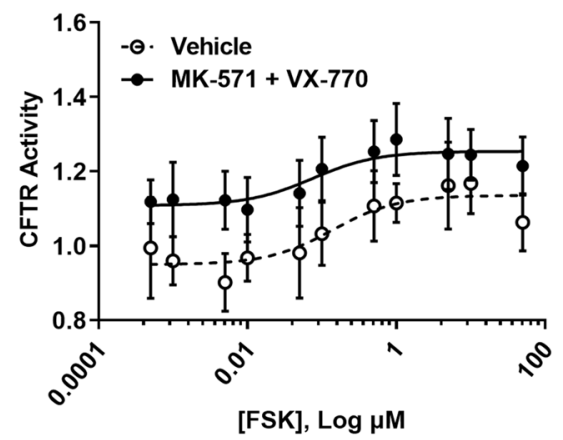

b

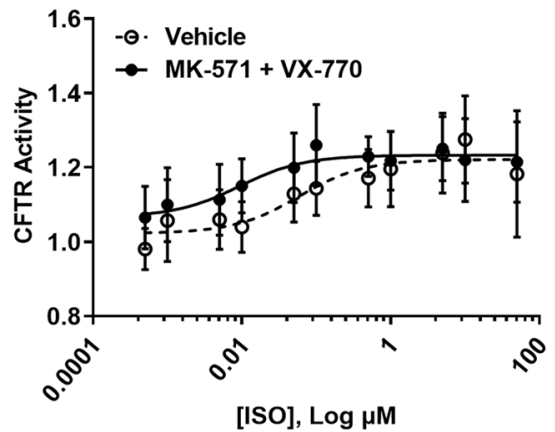

Roflimilast + VX-770

C
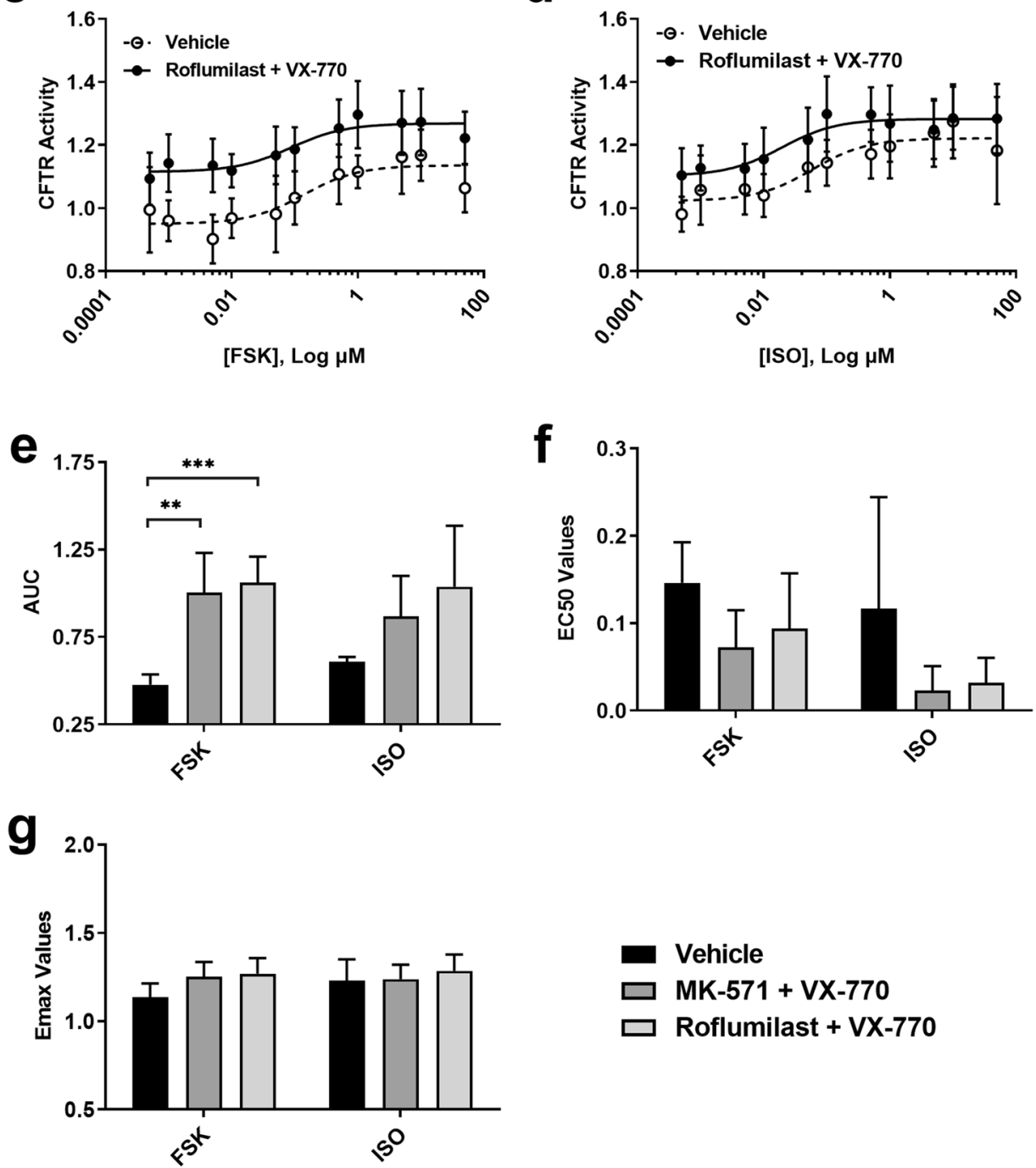

$\square$ Roflumilast + VX-770

Figure 7. Consequences of ABCC4 and PDE-4 inhibition on CFTR function in the presence of CFTR modulator VX-770 using receptor-independent (forskolin) and receptor-dependent (isoproterenol) cAMP inducers. ABCC4 and PDE4 were inhibited with MK-571 $(1.8 \mu \mathrm{M})$ and Roflumilast $(1 \mu \mathrm{M})$ respectively prior to VX-770 $(1 \mu \mathrm{M})$ stimulation. Forskolinstimulated (a,c) CFTR activity in the presence of (a) MK-571 + VX-770 and (c) Roflumilast + VX-770. Isoproterenolstimulated (b,d) CFTR activity in the presence of (b) MK-571 + VX-770 and (d) Roflumilast + VX-770. Analysis of concentration-response curves was performed for (e) AUC, (f) $\mathrm{EC}_{50}$, and (g) $\mathrm{E}_{\max }$. Each concentration-response curve was normalized to baseline over DMSO vehicle control. All data presented as means \pm standard deviations $(\mathrm{n}=6-7$, MK-571 + VX-770 and Roflumilast +VX-770). ${ }^{* *} P \leq 0.01 ;{ }^{* *} P \leq 0.001$. 
a

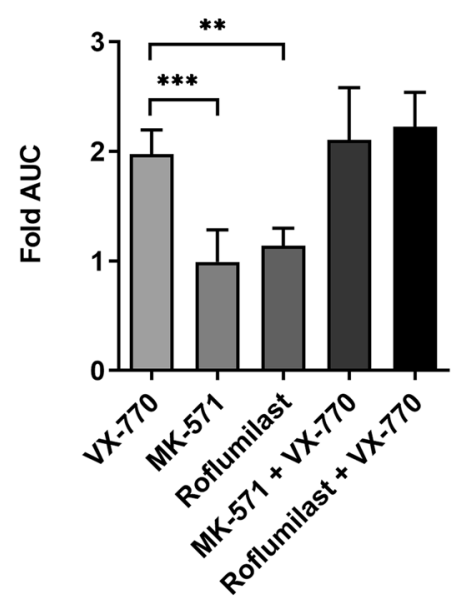

d

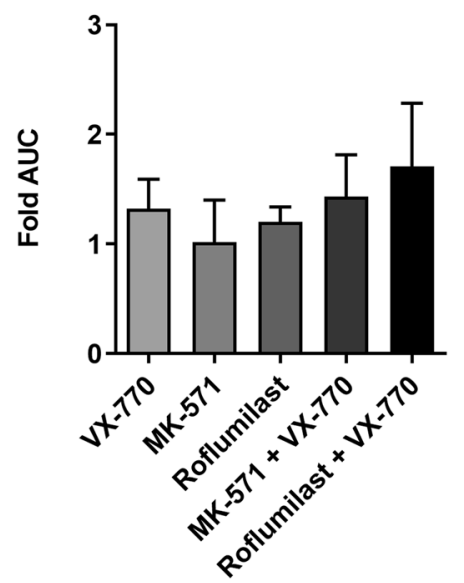

b

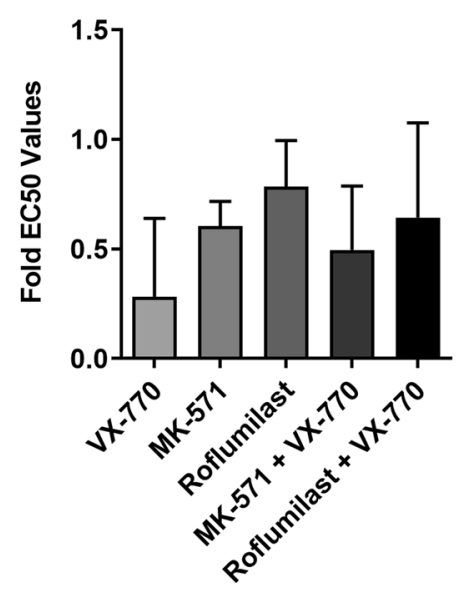

e

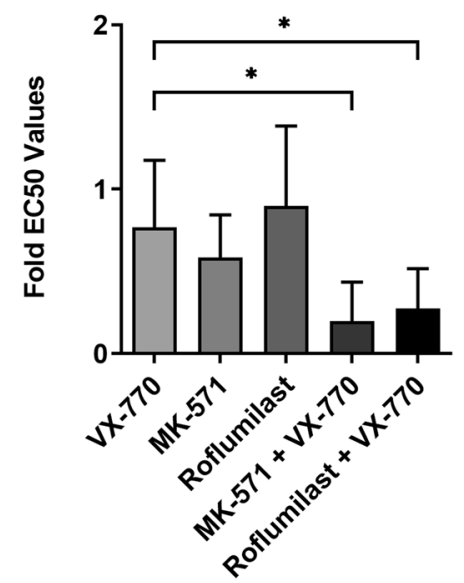

C

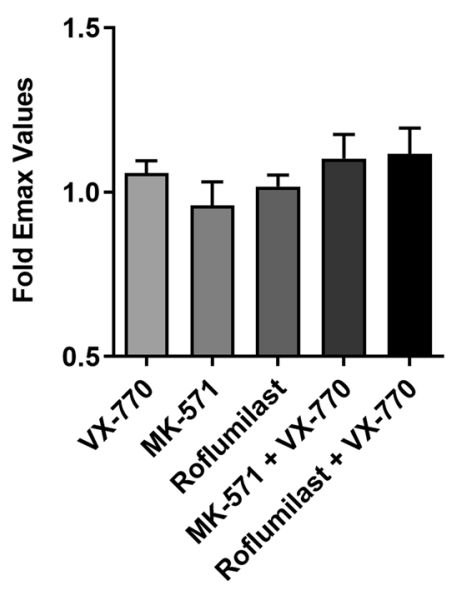

f

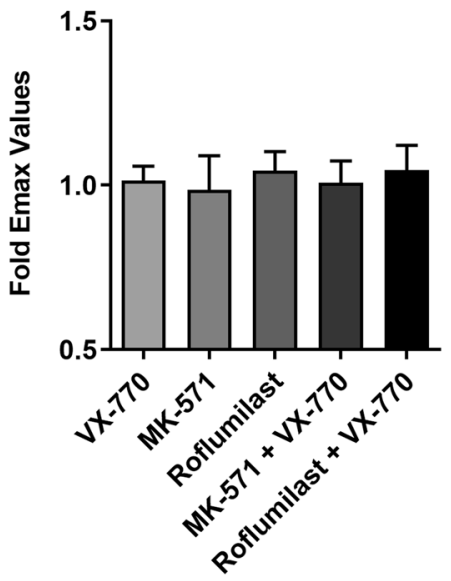

Figure 8. Fold-change comparisons between CFTR modulator VX-770, ABCC4 inhibition, and PDE-4 inhibition. A comparison between CFTR modulator VX-770, ABCC4 inhibitor MK-571, PDE-4 Inhibitor Roflumilast, and VX-770 \pm MK-571 or Roflumilast was performed using the values determined from their concentration-response curves in the presence of forskolin $(\mathbf{a}-\mathbf{c})$ or isoproterenol $(\mathbf{d}-\mathbf{f})$. Each treatment was normalized to its vehicle control to allow for comparison. A fold-change of (a,d) AUC, (b,e) $\mathrm{EC}_{50}$, and (c,f) $\mathrm{E}_{\max }$ are depicted. All data presented as means \pm standard deviations $(\mathrm{n}=4-8, \mathrm{VX}-770 ; \mathrm{n}=4-5, \mathrm{MK}-571 ; \mathrm{n}=5$, Roflumilast; $\mathrm{n}=6-7, \mathrm{MK}-571+\mathrm{VX}-770$ and Roflumilast $+\mathrm{VX}-770) .{ }^{\star} P \leq 0.05 ;{ }^{* \star} P \leq 0.01 ;{ }^{* \star} P \leq 0.001$.

Combinatorial approaches have been suggested as the future for modulation of CFTR activity and may include direct or indirect approaches ${ }^{13}$. Modulation of cAMP levels functions as an indirect mechanism to modulate CFTR and could potentially be combined with direct CFTR modulator approaches. We therefore decided to investigate ABCC4 and PDE-4 inhibitors which are able to differentially modulate cAMP levels by blocking extracellular transport and intracellular breakdown, respectively.

It has been previously demonstrated that pharmacological inhibition of ABCC4 by MK-571, in combination with VX-770, was able to improve CFTR activity in primary nasal epithelial cells from CF patients heterozygous for the G551D-variant ${ }^{14}$. In addition, in primary non-CF HBECs, it has been demonstrated that the administration of Roflumilast led to CFTR activation ${ }^{38}$. However, in this same study, the addition of Roflumilast to Calu-3 cells did not potentiate CFTR activity beyond the max stimulation that resulted from the addition of FSK ${ }^{38}$.

In our concentration-response analysis of ABCC4 and PDE-4 inhibitors in Calu-3 cells, only mild CFTR potentiation was observed alone or in combination with VX-770. Our results for ABCC4 inhibition contrast our previous demonstration of ABCC4 inhibitor MK-571 in potentiating CFTR activity in G551D-variant expressing primary human airway epithelial cells ${ }^{14}$. Conversely, our results of PDE-4 inhibitors align with previous findings in primary HBECs that were exposed to whole cigarette smoke in which Roflumilast, in combination with VX-770, demonstrated an increase in short-circuit current, suggesting this combination leads to an increase in CFTR activity $^{38}$. We highlight that it may be possible that wild-type CFTR expressed in Calu-3 cells is maximally active and difficult to further potentiate with cAMP modulating agents. Our data showing shifts in the left of the curve (increased sensitivity of CFTR activity) without changes in maximal response support this concept. Specifically, the addition of VX-770 with either cAMP inducer to Calu-3 cells led to an upward shift in the curve, 
a significant increase in AUC but no significant changes to $\mathrm{E}_{\max }$. However, VX-770 with FSK showed a significant decrease in $\mathrm{EC}_{50}$ and VX-770 with ISO showed a significant increase in baseline values. When VX-770 was combined with an ABCC4 or PDE-4 inhibitor and cAMP inducer FSK, there were significant increases in AUC, indicating an increase in CFTR activity, but again no change in $\mathrm{E}_{\max }$. In order to determine whether ABCC4 and PDE-4 inhibitor with VX-770 had an additive effect beyond VX-770 alone, a fold-change analysis for AUC, $\mathrm{EC}_{50}$, and $\mathrm{E}_{\max }$ values of the independent experiments was performed for indirect comparisons. Combinatorial additions of VX-770 with ABCC4 or PDE-4 inhibitor, in the presence of cAMP inducer ISO, led to a decrease in fold $\mathrm{EC}_{50}$ values compared to VX-770 alone, suggesting that ABCC4 and PDE-4 inhibitors may increase the sensitivity to VX-770. In addition, with cAMP inducer FSK, VX-770 with Roflumilast had a significant increase in fold baseline values, suggesting that there may also be an increase in efficacy. With these fold-change comparison analyses in mind, further investigation of these combinatorial treatments should be performed.

Our interrogation of the role of ABCC4 in airway epithelial cell modulation of cAMP levels is grounded in reports from primary human airway epithelial cells and cell lines, while examining CFTR activity in Calu-3 cells has a strong foundation $35,41,42,53,54$. To determine the optimal cell system to perform ABCC4 interventions with outcomes of CFTR activity, we interrogated both HBEC-6KT and Calu-3 cells for ABCC4 and CFTR protein expression levels. To our surprise, while ABCC4 is present in both cell lines, CFTR is only present in the Calu-3 cells, leading to the selection of Calu-3 cells as the model of study. Our observation highlights the importance of basal expression analysis for CFTR protein in any human airway epithelial cells or lines prior to performing functional experiments.

Elevations in intracellular cAMP can be induced via multiple G protein-coupled receptor-dependent ( $\beta$-adrenergic receptor and adenosine receptor agonists-ISO and adenosine respectively) and independent mechanisms (direct activation of the enzyme adenylyl cyclase-FSK) ${ }^{64-68}$. The compartmentalization of cAMP signalling may be important for downstream signalling pathways within the cell-with specific implications for CFTR activity ${ }^{34,69}$. To determine whether the mechanism of cAMP production impacts CFTR activity, Calu-3 cells were treated with FSK and ISO at varying concentrations. Sigmoidal shape concentration-response curves were observed for both cAMP-elevating agents with the maximal degree of change for CFTR activity greater with ISO. The interpretation of this observation is that the compartmentalization of cAMP to the plasma membrane may more effectively couple protein kinase A activation to CFTR phosphorylation, which could be masked by global cytosolic increases in cAMP induced by FSK $^{34,69}$.

In our previous studies, we have not performed complete concentration-response analysis experiments for the leukotriene receptor antagonist MK-571, which has off-target ABCC4 inhibition effects, in parallel to the more selective ABCC4 inhibitor Ceefourin-1, thus we wanted to explore both in greater detail ${ }^{14,42,45}$. In our concentration-response analysis experiments utilizing an in vitro extracellular cAMP assay platform, both commercially available Ceefourin-1 and MK-571 were able to decrease extracellular cAMP levels, thus both were used in downstream in vitro CFTR membrane potential assays to investigate the consequences of ABCC4 inhibition on CFTR function. Due to similar findings using either compound in the in vitro CFTR membrane potential assay and previous demonstrations of MK-571, in combination with VX-770, potentiating CFTR activity beyond VX-770 alone, MK-571 was selected for combinational studies ${ }^{14}$.

While our findings suggest that cAMP modulation has the potential to be used as an add-on therapy with existing therapeutics, limitations of the study should be noted. Although Calu-3 cells expressed both ABCC4 and CFTR, it might not have been an appropriate model of study due to its WT-CFTR expression. Potentiation of CFTR activity by ABCC4 or PDE-4 inhibitors in the literature were performed in primary cells, suggesting that a potential add-on combinatorial therapy may only show an effect in primary cell cultures, especially ones with CFTR defects. This emphasizes that further investigation of ABCC4 and PDE-4 inhibitors as a potential add-on combinatorial therapy should be performed in primary human airway epithelial cells from a large subset of CF subjects, covering a wide-range of CFTR variants with compromised CFTR expression or function, in order to determine which patient populations could benefit from cAMP modulation therapy. In addition, further investigations of cAMP modulation with other drugs that modulate CFTR, such as VX-770, VX-809, VX-661, VX-445, and genistein, should be tested as it may be more efficacious.

\section{Methods}

Reagents. The human bronchial epithelial cell (HBEC6-KT) line was cultured in Keratinocyte Serum-Free Medium 1X (Gibco) according to the manufacturers directions and supplemented with P/S (VWR) at $1 \mathrm{X}^{70}$. Human airway epithelial cell line Calu-3 (ATCC HTB-55) were cultured in Minimum Essential Medium Alpha Medium (Corning) and supplemented with Premium Grade FBS (VWR) at 10\%, HEPES (Corning) at 1X, and $\mathrm{P} / \mathrm{S}$ (VWR) at 1X. The following chemical reagents were dissolved in DMSO, cAMP elevating agents Forskolin (Cayman Chemical) and Isoproterenol (Cayman Chemical), ABCC4 inhibitors MK-571 (Cayman Chemical) and Ceefourin 1 (Abcam), PDE inhibitors Roflumilast (Cayman Chemical) and Rolipram (Cayman Chemical), and CFTR Inhibitor (Selleck Chemicals).

Human airway epithelial cell lines. Two human airway epithelial cell lines were used for in vitro experiments. HBEC6-KT cells, provided by Dr. John Minna and Dr. Darryl Knight, are derived from healthy nonsmoking individuals and immortalized through human telomerase reverse transcriptase and cyclin-dependent kinase 4 expression was used for in vitro concentration-response analysis of ABCC4 inhibitors through an extracellular cAMP assay ${ }^{41,42,70-72}$. Calu-3 cells derived from the metastatic site of lung adenocarcinoma tissue was used for both in vitro concentration-response analysis of ABCC4 inhibitors and in vitro analysis of CFTR function. 
ABCC4 and CFTR protein expression analysis. HBEC6-KT and Calu-3 cells were lysed using RIPA Lysis Buffer containing Protease Inhibitor Cocktail powder (Sigma-Aldrich) for $60-90 \mathrm{~min}$ at $4{ }^{\circ} \mathrm{C}$ on a rocker. Lysates were then centrifuged at $16,000 \mathrm{xg}$ for $15 \mathrm{~min}$ with the supernatants collected for downstream immunoblots for ABCC4 and CFTR. Protein quantification was performed using a BCA protein assay. Twenty micrograms of protein were loaded on 4-15\% gradient TGX Stain-Free Protein Gels and transferred to Immun-Blot LF PVDF membrane (Bio-Rad). The membranes were blocked with $1 \mathrm{X}$ TBS with $0.05 \%$ Tween 20 and $5 \%$ skim milk powder for $2 \mathrm{~h}$ at $25^{\circ} \mathrm{C}$. The membranes were then incubated with primary antibody ABCC4/MRP4 (1:40, Abcam, AB15602) or CFTR (1:5000, UNC-Chapel Hill, AB596) overnight. The membranes were then washed in 1X TBS with $0.05 \%$ Tween 20 and incubated with HRP-linked anti-rat secondary antibody (1:3000, Cell Signaling Technology, 7077S) or anti-mouse secondary antibody (1:3000, Cell Signaling Technology, 7076S) for $2 \mathrm{~h}$ at $25^{\circ} \mathrm{C}$. A chemiluminescence image of the blot was taken using the Bio-Rad Image Lab software. Full western blot images have been included and can be found in the supplementary information (Supplementary Fig. 4).

In vitro extracellular cAMP assay. Calu-3 and HBEC6-KT cells were cultured as described previously $y^{41,42,71,72}$. Cells were pretreated with IBMX $(20 \mu \mathrm{M})$ for $2 \mathrm{~h}$ prior to experimental conditions. After the pre-treatment of IBMX, cells were treated with ABCC4 inhibitors (0.01-100 $\mu \mathrm{M})$ or DMSO for $30 \mathrm{~min}$. Following these exposures, the cells were treated with forskolin $(10 \mu \mathrm{M})$ for $6 \mathrm{~h}$ and cell-culture supernatants were collected for analysis of extracellular cAMP. The negative control was IBMX alone and the positive control was IBMX and forskolin.

In vitro CFTR membrane potential assay. An in vitro CFTR membrane potential assay was performed on Calu-3 cells and primary human bronchial epithelial cells, from two independent donors, cultured at $37^{\circ} \mathrm{C}$ as previously described ${ }^{14}$. All human samples were collected from consented individuals under ethics approval granted by Hamilton Integrated Research Ethics Board (Project Number 5099-T).

Calu-3 cells were grown for 21 days on 96-well plates and primary HBECs were grown for 14 days under air-liquid interface conditions on collagen-coated (Sigma-Aldrich) $6.5 \mathrm{~mm}$ Transwell Inserts (Corning). All cells were washed with HBSS prior to experimental conditions. After washing with HBSS, cells were loaded with BLUE Membrane Potential Dye (Molecular Devices, \#R8042) dissolved in $37^{\circ} \mathrm{C}$ chloride-free buffer (NMDG Gluconate Buffer - 150 mM NMDG-gluconate, 3 mM KCl, 10 mM HEPES, pH 7.35, 300 mOsm). Immediately after dye loading, CFTR activity measurements were taken. CFTR activity measurements are fluorescent readouts for detecting ion channel activity. CFTR inhibition was performed with CFTRinh-172 (10 $\mu \mathrm{M})$ for all experiments to attribute changes in fluorescence as CFTR channel activity. This assay has been validated using proof of concept studies where results obtained from this assay were able to recapitulate findings obtained from direct measurements of ion channel activity using Ussing chambers ${ }^{14}$. At the end of this assay, raw data was exported for statistical analysis. Tracings demonstrating the variation in CFTR Activity as a function of time, for key experiments, have been included in the supplementary information (Supplementary Fig. 5).

For Calu-3 cells, measurements were taken during baseline (40 $\mathrm{min})$, cAMP elevation (30 min), and CFTR inhibition $(20 \mathrm{~min})$. cAMP elevation was performed using forskolin or isoproterenol $(0.0005-50 \mu \mathrm{M})$. For assays with ABCC4 or PDE-4 inhibitors, a pre-incubation with the inhibitors (30 $\mathrm{min}$ ) in the plate reader was performed prior to cAMP elevation. CFTR modulator VX-770 was used at $1 \mu \mathrm{M}$. CFTR activity was determined by dividing a single membrane potential peak measurement after the ABCC4 or PDE-4 inhibition and cAMP elevation additions to the stabilized baseline over DMSO control.

For primary HBECs, measurements were taken during baseline (8 min), drug additions (40 min), and CFTR inhibitions (18 $\mathrm{min})$. cAMP elevation was performed using forskolin $(10 \mu \mathrm{M})$. The same concentrations used in Calu-3 cells for ABCC4 and PDE4 inhibitors, and VX-770 were used for primary HBECs, however the preincubation was skipped and instead, all drug combinations were added once at the beginning of the drug addition step. CFTR activity was determined by averaging six independent measurements within a single Transwell insert. Measurements were normalized to the averaged baseline over DMSO control.

Statistical analysis. For the in vitro extracellular cAMP assay, values were normalized to the positive control (IBMX and forskolin) and the half maximal inhibitory concentration $\left(\mathrm{IC}_{50}\right)$ was determined. SD was calculated using data from biological replicates $(n=4-5)$.

For in vitro CFTR membrane potential assays, in Calu-3 cells, values were normalized to the averaged baseline over averaged vehicle (DMSO) control of all biological replicates. SD was calculated using data from biological replicates $(n=4-8)$. An area under the curve (AUC), half-maximal concentration $\left(\mathrm{EC}_{50}\right)$, maximum concentration $\left(\mathrm{E}_{\max }\right)$, and baseline analysis was performed for each individual replicate then averaged together. The AUC analysis is an aggregate measure of sensitivity and maximal response, allowing for the observation of the total net responsiveness of the cells. The AUC analysis was done by normalizing the individual replicates to the bottom most value of the averaged vehicle control. In primary HBECs, six independent measurements were taken within a single Transwell insert. The value is the average of at least six measurements. These values were normalized to the averaged baseline over the averaged vehicle (DMSO) control. SD was calculated by treating the six measurements as independent, with additional technical replicates $(n=6-18)$. An AUC and max peak analysis were performed for each measurement, then averaged together. The max peak analysis was the max CFTR activity achieved during the drug addition step.

In order to compare the independent experiments performed, a fold-change analysis using individual AUC, $\mathrm{EC}_{50}$, and $\mathrm{E}_{\max }$, determined from their respective concentration-response curves, was performed and was normalized to the averaged DMSO vehicle control. A one-way ANOVA with subsequent post-hoc test or a paired $t$-test was performed. Statistical analysis was performed using GraphPad Prism 6. 


\section{Data availability}

The datasets generated and analysed during the course of the study are available from the corresponding author on reasonable request.

Received: 5 June 2020; Accepted: 7 December 2020

Published online: 13 January 2021

\section{References}

1. Riordan, J. R. et al. Identification of the cystic fibrosis gene: Cloning and characterization of complementary DNA. Science (80-. ). 245, 1066-1073 (1989).

2. Anderson, M. P. et al. Demonstration that CFTR is a chloride channel by alteration of its anion selectivity. Science (80-. ). 253, 202-205 (1991).

3. Bear, C. E. et al. Purification and functional reconstitution of the cystic fibrosis transmembrane conductance regulator (CFTR). Cell 68, 809-818 (1992).

4. Smith, J. J. \& Welsh, M. J. cAMP stimulates bicarbonate secretion across normal, but not cystic fibrosis airway epithelia. J. Clin. Invest. 89, 1148-1153 (1992).

5. Saint-Criq, V. \& Gray, M. A. Role of CFTR in epithelial physiology. Cell. Mol. Life Sci. 74, 93-115 (2017).

6. Jayaraman, S., Joo, N. S., Reitz, B., Wine, J. J. \& Verkman, A. S. Submucosal gland secretions in airways from cystic fibrosis patients have normal $[\mathrm{Na}(+)]$ and $\mathrm{pH}$ but elevated viscosity. Proc. Natl. Acad. Sci. U. S. A. 98, 8119-8123 (2001).

7. Tang, X. X. et al. Acidic pH increases airway surface liquid viscosity in cystic fibrosis. J. Clin. Invest. 126, 879-891 (2016).

8. Pezzulo, A. A. et al. Reduced airway surface pH impairs bacterial killing in the porcine cystic fibrosis lung. Nature 487, 109-113 (2012).

9. Alaiwa, M. H. A. et al. $\mathrm{pH}$ modulates the activity and synergism of the airway surface liquid antimicrobials $\beta$-defensin-3 and LL-37. Proc. Natl. Acad. Sci. U. S. A. 111, 18703-18708 (2014).

10. Welsh, M. J. \& Smith, A. E. Molecular mechanisms of CFTR chloride channel dysfunction in cystic fibrosis. Cell 73, 1251-1254 (1993).

11. Zielenski, J. \& Tsui, L.-C. Cystic Fibrosis: Genotypic and Phenotypic Variations. Annu. Rev. Genet. 29, 777-807 (1995).

12. Rowe, S. M., Miller, S. \& Sorscher, E. J. Cystic fibrosis. N. Engl. J. Med. 352, 1992-2001 (2005).

13. Veit, G. et al. From CFTR biology toward combinatorial pharmacotherapy: Expanded classification of cystic fibrosis mutations. Mol. Biol. Cell 27, 424-433 (2016).

14. Ahmadi, S. et al. Phenotypic profiling of CFTR modulators in patient-derived respiratory epithelia. npj Genomic Med. 2, 1-10 (2017).

15. KALYDECO (ivacaftor) Package Insert. (Vertex Pharmaceuticals Incorporated, Boston, 2019).

16. ORKAMBI (lumacaftor/ivacaftor) Package Insert. (Vertex Pharmaceuticals Incorporated, Boston, 2019).

17. SYMDEKO (tezacaftor/ivacaftor) Package Insert. (Vertex Pharmaceuticals Incorporated, Boston, 2019).

18. TRIKAFTA (elexacaftor, tezacaftor and ivacaftor) Package Insert. (Vertex Pharmaceuticals Incorporated, Boston, 2020).

19. Van Goor, F. et al. Rescue of CF airway epithelial cell function in vitro by a CFTR potentiator, VX-770. Proc. Natl. Acad. Sci. U. S. A. 106, 18825-18830 (2009).

20. Accurso, F. J. et al. Effect of VX-770 in persons with cystic fibrosis and the G551D-CFTR mutation. N. Engl. J. Med. 363, 1991-2003 (2010).

21. Ramsey, B. W. et al. A CFTR potentiator in patients with cystic fibrosis and the G551D mutation. N. Engl. J. Med. 365, 1663-1672 (2011).

22. Van Goor, F. et al. Correction of the F508del-CFTR protein processing defect in vitro by the investigational drug VX-809. Proc. Natl. Acad. Sci. U. S. A. 108, 18843-18848 (2011).

23. Boyle, M. P. et al. A CFTR corrector (lumacaftor) and a CFTR potentiator (ivacaftor) for treatment of patients with cystic fibrosis who have a phe508del CFTR mutation: a phase 2 randomised controlled trial. Lancet. Respir. Med. 2, 527-538 (2014).

24. Wainwright, C. E., Elborn, J. S. \& Ramsey, B. W. Lumacaftor-Ivacaftor in patients with cystic fibrosis homozygous for Phe508del CFTR. N. Engl. J. Med. 373, 1783-1784 (2015).

25. Taylor-Cousar, J. L. et al. Tezacaftor-ivacaftor in patients with cystic fibrosis homozygous for Phe508del. N. Engl. J. Med. 377, 2013-2023 (2017).

26. Rowe, S. M. et al. Tezacaftor-ivacaftor in residual-function heterozygotes with cystic fibrosis. N. Engl. J. Med. 377, 2024-2035 (2017).

27. Keating, D. et al. VX-445-tezacaftor-ivacaftor in patients with cystic fibrosis and one or two Phe508del alleles. N. Engl. J. Med. 379, $1612-1620$ (2018).

28. Middleton, P. G. et al. Elexacaftor-tezacaftor-ivacaftor for cystic fibrosis with a single Phe508del allele. N. Engl. J. Med. 381, 1809-1819 (2019).

29. Davies, J. C. et al. VX-659-tezacaftor-ivacaftor in patients with cystic fibrosis and one or two Phe508del alleles. N. Engl. J. Med. 379, 1599-1611 (2018)

30. Bell, S. C. et al. CFTR activity is enhanced by the novel corrector GLPG2222, given with and without ivacaftor in two randomized trials. J. Cyst. Fibros. 18, 700-707 (2019).

31. Cheng, S. H. et al. Phosphorylation of the R domain by cAMP-dependent protein kinase regulates the CFTR chloride channel. Cell 66, 1027-1036 (1991).

32. Picciotto, M. R., Cohn, J. A., Bertuzzi, G., Greengard, P. \& Nairn, A. C. Phosphorylation of the cystic fibrosis transmembrane conductance regulator. J. Biol. Chem. 267, 12742-12752 (1992).

33. Winter, M. C. \& Welsh, M. J. Stimulation of CFTR activity by its phosphorylated R domain. Nature 389, 294-296 (1997).

34. Li, C. et al. Spatiotemporal coupling of cAMP transporter to CFTR chloride channel function in the gut epithelia. Cell 131, 940-951 (2007).

35. Conner, G. E., Ivonnet, P., Gelin, M., Whitney, P. \& Salathe, M. HO Stimulates CFTR through an autocrine prostaglandin pathway using MRP4. Am. J. Respir. Cell Mol. Biol. 49, 672-679 (2013).

36. Cobb, B. R., Fan, L., Kovacs, T. E., Sorscher, E. J. \& Clancy, J. P. Adenosine receptors and phosphodiesterase inhibitors stimulate Cl - secretion in Calu-3 cells. Am. J. Respir. Cell Mol. Biol. 29, 410-418 (2003).

37. Blanchard, E. et al. Anchored PDE4 regulates chloride conductance in wild-type and $\triangle \mathrm{f} 508$-CFTR human airway epithelia. FASEB J. 28, 791-801 (2014).

38. Lambert, J. A. et al. Cystic fibrosis transmembrane conductance regulator activation by roflumilast contributes to therapeutic benefit in chronic bronchitis. Am. J. Respir. Cell Mol. Biol. 50, 549-558 (2014).

39. Naren, A. P. et al. A macromolecular complex of $\beta 2$ adrenergic receptor, CFTR, and ezrin/radixin/moesin-binding phosphoprotein 50 is regulated by PKA. Proc. Natl. Acad. Sci. U. S. A. 100, 342-346 (2003).

40. Schuetz, J. D. et al. MRP4: A previously unidentified factor in resistance to nucleoside-based antiviral drugs. Nat. Med. 5, 1048-1051 (1999). 
41. Gold, M. J. et al. Mucosal production of uric acid by airway epithelial cells contributes to particulate matter-induced allergic sensitization. Mucosal Immunol. 9, 809-820 (2016).

42. Huff, R. D. et al. Inhibition of ABCC4 potentiates combination beta agonist and glucocorticoid responses in human airway epithelial cells. J. Allergy Clin. Immunol. 141, 1127-1130.e5 (2018).

43. Turner, M. J. et al. The dual phosphodiesterase 3 and 4 inhibitor RPL554 stimulates CFTR and ciliary beating in primary cultures of bronchial epithelia. Am. J. Physiol. - Lung Cell. Mol. Physiol. 310, L59-L70 (2016).

44. Kips, J. C. et al. MK-571, a potent antagonist of leukotriene D4-induced bronchoconstriction in the human. Am. Rev. Respir. Dis. https://doi.org/10.1164/ajrccm/144.3_pt_1.617 (1991).

45. Cheung, L. et al. High-throughput screening identifies Ceefourin 1 and Ceefourin 2 as highly selective inhibitors of multidrug resistance protein 4 (MRP4). Biochem. Pharmacol. 91, 97-108 (2014).

46. Calverley, P. M. et al. Roflumilast in symptomatic chronic obstructive pulmonary disease: Two randomised clinical trials. Lancet 374, 685-694 (2009).

47. Fabbri, L. M. et al. Roflumilast in moderate-to-severe chronic obstructive pulmonary disease treated with longacting bronchodilators: two randomised clinical trials. Lancet 374, 695-703 (2009).

48. Martinez, F. J. et al. Effect of roflumilast on exacerbations in patients with severe chronic obstructive pulmonary disease uncontrolled by combination therapy (REACT): A multicentre randomised controlled trial. Lancet 385, 857-866 (2015).

49. Criner, G. J. et al. Prevention of acute exacerbations of COPD: American College of Chest Physicians and Canadian Thoracic Society Guideline. Chest 147, 894-942 (2015).

50. Wachtel, H. Potential antidepressant activity of rolipram and other selective cyclic adenosine $3^{\prime}, 5^{\prime}$-monophosphate phosphodiesterase inhibitors. Neuropharmacology 22, 267-272 (1983).

51. Zhu, J., Mix, E. \& Winblad, B. The antidepressant and antiinflammatory effects of rolipram in the central nervous system. CNS Drug Rev. 7, 387-398 (2001).

52. O'Donnell, J. M. \& Zhang, H. T. Antidepressant effects of inhibitors of cAMP phosphodiesterase (PDE4). Trends Pharmacol. Sci. 25, 158-163 (2004).

53. Shen, B. Q., Finkbeiner, W. E., Wine, J. J., Mrsny, R. J. \& Widdicombe, J. H. Calu-3: A human airway epithelial cell line that shows cAMP-dependent Cl- secretion. Am. J. Physiol.-Lung Cell. Mol. Physiol. 266 (1994).

54. Haws, C., Finkbeiner, W. E., Widdicombe, J. H. \& Wine, J. J. CFTR in Calu-3 human airway cells: Channel properties and role in cAMP-activated Cl-conductance. Am. J. Physiol. Lung Cell. Mol. Physiol. 266 (1994).

55. Varga, K. et al. Efficient intracellular processing of the endogenous cystic fibrosis transmembrane conductance regulator in epithelial cell lines. J. Biol. Chem. 279, 22578-22584 (2004).

56. Mathias, N. R. et al. Permeability characteristics of Calu-3 human bronchial epithelial cells: In vitro-in vitro correlation to predict lung absorption in rats. J. Drug Target. 10, 31-40 (2002).

57. Grainger, C. I., Greenwell, L. L., Lockley, D. J., Martin, G. P. \& Forbes, B. Culture of Calu-3 cells at the air interface provides a representative model of the airway epithelial barrier. Pharm. Res. 23, 1482-1490 (2006).

58. Haghi, M. et al. Time- and passage-dependent characteristics of a Calu-3 respiratory epithelial cell model. Drug Dev. Ind. Pharm. 36, 1207-1214 (2010).

59. Litosch, I., Hudson, T. H., Mills, I. \& Fain, J. N. Forskolin an an activator of cyclic AMP accumulation and lipolysis in rat adipocytes. Mol. Pharmacol. 22, 109-115 (1982).

60. Vegesna, R. V. K. \& Diamond, J. Comparison of the effects of forskolin and isoproterenol on cyclic AMP levels and tension in bovine coronary artery. Can. J. Physiol. Pharmacol. 61, 1202-1205 (1983).

61. Buxton, I. L. O. \& Brunton, L. L. Compartments of cyclic AMP and protein kinase in mammalian cardiomyocytes. J. Biol. Chem. 258, 10233-10239 (1983).

62. Zhou, H.-L., Newsholme, S. J. \& Torphy, T. J. Agonist-related differences in the relationship between cAMP content and protein kinase activity in canine trachealis. J. Pharmacol. Exp. Ther. 261, 1260-1267 (1992).

63. Reenstra, W. W., Yurko-Mauro, K., Dam, A., Raman, S. \& Shorten, S. CFTR chloride channel activation by genistein: The role of serine/threonine protein phosphatases. Am. J. Physiol. Cell Physiol. 271 (1996).

64. Serezani, C. H., Ballinger, M. N., Aronoff, D. M. \& Peters-Golden, M. Cyclic AMP: Master regulator of innate immune cell function. Am. J. Respir. Cell Mol. Biol. 39, 127-132 (2008).

65. Seamon, K. B., Padgett, W. \& Daly, J. W. Forskolin: Unique diterpene activator of adenylate cyclase in membranes and in intact cells. Proc. Natl. Acad. Sci. U. S. A. 78, 3363-3367 (1981).

66. Huang, P. et al. Compartmentalized autocrine signaling to cystic fibrosis transmembrane conductance regulator at the apical membrane of airway epithelial cells. Proc. Natl. Acad. Sci. U. S. A. 98, 14120-14125 (2001).

67. Feoktistov, I. \& Biaggioni, I. Adenosine A2B receptors. Pharmacol. Rev. 49, 381-402 (1997).

68. Gilman, A. G. G proteins: Transducers of receptor-generated signals. Annu. Rev. Biochem. 56, 615-649 (1987).

69. Cheepala, S. et al. Cyclic nucleotide compartmentalization: Contributions of phosphodiesterases and ATP-binding cassette transporters. Annu. Rev. Pharmacol. Toxicol. 53, 231-253 (2013).

70. Ramirez, R. D. et al. Immortalization of human bronchial epithelial cells in the absence of viral oncoproteins. Cancer Res. 64, 9027-9034 (2004).

71. Hirota, J. A. et al. The nucleotide-binding domain, leucine-rich repeat protein 3 inflammasome/IL-1 receptor I axis mediates innate, but not adaptive, immune responses after exposure to particulate matter under $10 \mu \mathrm{m}$. Am. J. Respir. Cell Mol. Biol. 52, 96-105 (2015).

72. Huff, R. D. et al. Regulation of xanthine dehydrogensase gene expression and uric acid production in human airway epithelial cells. PLoS One 12 (2017).

\section{Acknowledgements}

This research was supported by funding from the Canadian Institutes of Health Research (CIHR).

\section{Author contributions}

J.P.N. performed in vitro CFTR membrane potential assay experiments, data analyses, figure generation, and literature review; contributed to the manuscript conception; and drafted the manuscript. M.B. performed in vitro CFTR membrane potential assay experiments. R.D.H.. performed in vitro extracellular cAMP assay experiments, data analyses, and figure generation. N.T. performed western blotting and figure generation. M.D.I. contributed to the manuscript conception. J.A.H. (Principle Investigator and corresponding author) was responsible for the oversight of the entire study (data collection, analysis, drafting, finalization) and supervision of trainees.

\section{Competing interests}

The authors declare no competing interests. 


\section{Additional information}

Supplementary Information The online version contains supplementary material available at https://doi. org/10.1038/s41598-020-79555-w.

Correspondence and requests for materials should be addressed to J.A.H.

Reprints and permissions information is available at www.nature.com/reprints.

Publisher's note Springer Nature remains neutral with regard to jurisdictional claims in published maps and institutional affiliations.

(c) (1) Open Access This article is licensed under a Creative Commons Attribution 4.0 International cc) License, which permits use, sharing, adaptation, distribution and reproduction in any medium or format, as long as you give appropriate credit to the original author(s) and the source, provide a link to the Creative Commons licence, and indicate if changes were made. The images or other third party material in this article are included in the article's Creative Commons licence, unless indicated otherwise in a credit line to the material. If material is not included in the article's Creative Commons licence and your intended use is not permitted by statutory regulation or exceeds the permitted use, you will need to obtain permission directly from the copyright holder. To view a copy of this licence, visit http://creativecommons.org/licenses/by/4.0/.

(C) The Author(s) 2021 